\title{
Analysis of anisotropic formations with the electromagnetic triaxial induction tool.
}

Paulo Roberto de Carvalho* (ICIBE/UFRA), Cícero Roberto Teixeira Régis (CPGf/UFPA)

Copyright 2016, SBGf - Sociedade Brasileira de Geofísica

Este texto foi preparado para a apresentação no VII Simpósio Brasileiro de Geofísica, Ouro Preto, 25 a 27 de outubro de 2016. Seu conteúdo foi revisado pelo Comitê Técnico do VII SimBGf mas não necessariamente representa a opinião da SBGf ou de seus associados. É proibida a reprodução total ou parcial deste material para propósitos comerciais sem prévia autorização da SBGf.

\begin{abstract}
We show some numerical responses for the triaxial or multicomponent induction tool to one-dimensional (1D) layered anisotropic geoelectric models, in which the presence of the borehole and the invasion zones are neglected, to simulate geological environments of hydrocarbon reservoirs with intrinsic and macroscopic electrical anisotropy.
\end{abstract}

We ignore the six cross-coupled components because our models have azimuthal symmetry which these signals are nulls. Thus, we modeled only the responses obtained from the coaxial and coplanar coils arrays.

The results show a strong sensitivity of the coplanar array to skin effect and above all a good sensitivity to anisotropy of the formations.

Our goals are to compare the responses with those from isotropic finely laminated zones and to perform a quantitative analysis of how well an intrinsic anisotropic layer can represent such environments within a chosen tolerance.

\section{Introduction}

With the progressive exploitation of the main hydrocarbon (HC) reservoirs, it becomes a necessity to turn the attention to smaller and more complex reservoirs, which are usually made of relatively thin layers, but may have good permeability and porosity. Often, these deposits have good economic potential for having alternately source and reservoir rocks, besides having a large enough lateral extension to accumulate a considerable amount of $\mathrm{HC}$ (oil and gas).

Before the year 2000, finely laminated reservoirs were underestimated or even ignored due to the geometric configuration of the coils in the traditional borehole logging tools by electromagnetic induction (EM), because, since their invention (Doll, 1945), the coil arrangement was coaxial to the well axis and provided an overestimated estimation of the conductivity in environments where the conductive laminae of shale mask the presence of the resistive laminae of sand, saturated in oil (Carvalho \& Verma, 1998).
From 2000 on, EM induction probes consist basically of a combination of a coaxial arrangement with two coplanar arrangements of coils, i.e., three sources and three sensors, with axes orthogonal to each other, and are thus commercially referred to as triaxial or multicomponent induction tool (Krigshäuser et al., 2000). These probes were designed originally to investigate thinly laminated reservoirs, and consequently, a strong anisotropic behavior. The responses of the various arrangements of coils are simultaneously registered on multiple channels at multiple frequencies and source-sensor spacing. (Figure 1).

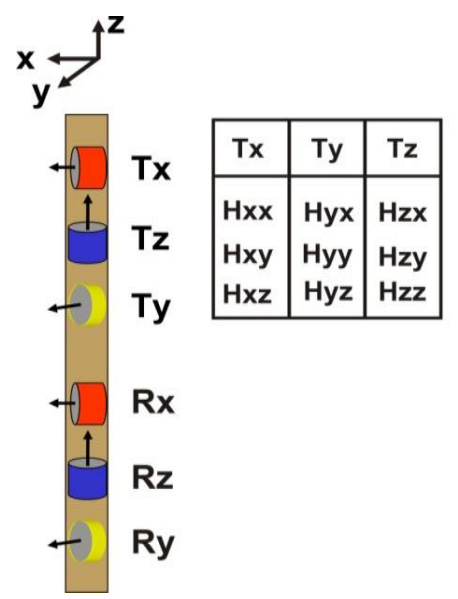

Figure 1 - Triaxial tool with a coaxial and two coplanar coils. arrays resulting in nine components of induced magnetic fields.

Currently, besides being the main location tool of finely laminated reservoirs (macroscopic anisotropy), triaxial probes are also applied in many situations of asymmetric geometry, such as locating dissolution cavities (vugs) and fractured zones in the vicinity of the wells, monitoring invasion fronts in horizontal wells, among others (Omeragic et al, 2015).

In this paper, we show some numerical responses for one-dimensional (1D) layered anisotropic geoelectric models, in which the presence of the borehole and the invasion zones are neglected, to simulate geological environments of hydrocarbon reservoirs with intrinsic electrical anisotropy.

We ignore the six cross-coupled components because our models have azimuthal symmetry which these signals are nulls (Ellis \& Singer, 2007). Thus, we modeled only the responses obtained from the coaxial and coplanar coil arrays, this leaves us with two different components of the 
magnetic field: $H_{z z}$ to the coaxial and $H_{x x}=H_{y y}$ to the coplanar responses.

Our goals are to compare the responses with those from isotropic finely laminated zones and to perform a quantitative analysis of how well an intrinsic anisotropic layer can represent such environments.

\section{Method}

In the theoretical treatment, the coils are represented as point magnetic dipoles since the radii of real coils in the induction tool are considered very small compared to the coil spacing and the wavelengths (Carvalho, et al., 2012).

The fields vary as $e^{i \omega t}$ where $i=\sqrt{-1} ; \omega=2 \pi f$ the angular frequency and $f$ the linear frequency. They are calculated in the quasi-static approximation $\left(\omega \varepsilon_{0} \ll \sigma\right)$ by use of magnetic and electric vector potentials, which are associated with the transverse magnetic (TM) and electric (TE) polarization modes of propagation, where $\varepsilon_{o}$ is the dielectric permittivity of free space and $\sigma$ the electric conductivity of the media. By applying appropriate boundary conditions to the fields on the interfaces between homogeneous regions, the surface impedances and the magnetic field components are calculated for vertical magnetic dipole (VMD) and horizontal magnetic dipole (HMD) sources.

In a basic two-coil array, the source produces only a single component on the axis of the receiver coil: $T_{z} / R_{z}$ for the coaxial and $T_{x} / R_{x}$ for the coplanar array. The raw apparent conductivity $\left(\sigma_{R}\right)$ is calculated from the in-phase with the transmitter current component of the voltage induced in the receiver coil (resistive signal).

The semi-analytical responses of the 1D models result in improper integrals due to the inverse Hankel transform, which are solved numerically with a 21 point GaussKronrod quadrature rule.

The main difference between the two models with anisotropic behavior simulated in this work is in the form of representing the electrical conductivity: 1) In the laminate package, consisting of thin isotropic layers of alternating sandstones $\left(\sigma_{s d}\right)$ and shales $\left(\sigma_{s h}\right)$ of the same thickness, the conductivities are scalar values. 2) In an intrinsically anisotropic layer, the conductivity is represented by a $3 \times 3$ tensor $\tilde{\sigma}$.

In Transversely Isotropic layers with a Vertical axis of symmetry (TIV), in which the main anisotropy directions are the same as the coordinate axes, the conductivity tensor reduces to

$\tilde{\sigma}=\left[\begin{array}{ccc}\sigma_{h} & 0 & 0 \\ 0 & \sigma_{h} & 0 \\ 0 & 0 & \sigma_{v}\end{array}\right]$.

This type of anisotropic medium has a characteristic parameter named coefficient of anisotropy, defined as

$$
\lambda^{2}=\sigma_{h} / \sigma_{v} \text {. }
$$

Following Kaufman \& Dashevsky (2010), for an extreme situation with sufficiently thin laminae, the expressions that correlate conductivities of the second model, with intrinsic anisotropy, and the first model, with macroscopic anisotropy are:

$\sigma_{h}=\frac{\left(\sigma_{s d}+\sigma_{s h}\right)}{2} \quad$ and $\quad \sigma_{v}=\frac{2\left(\sigma_{s d} \sigma_{s h}\right)}{\left(\sigma_{s d}+\sigma_{s h}\right)}$.

Thus, it is possible to find a laminae thickness below which the first model (macroscopic anisotropy) starts to give the same response as the second model (intrinsic anisotropy) within a chosen tolerance.

When the source and receiver coil axes are both oriented perpendicular to the bedding planes, as in the case of the coaxial array, only the component of the conductivity parallel to the planes affects the response. On the other hand, when both coils have axes oriented parallel to the bedding planes, as in the case of a coplanar array, the vertical conductivity affects significantly the response.

We can see in Figure 2 a schematic representation of the induced current generated by coaxial and coplanar arrays, in a well that was drilled perpendicular to the bedding in a thinly laminated formation.
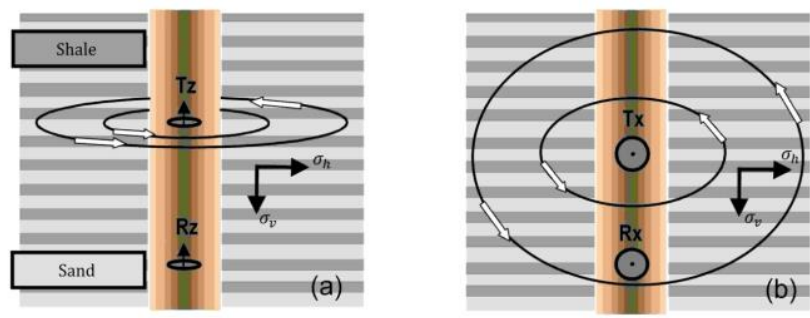

Figure 2 - Induction currents generated by the traditional coaxial coil array (a) and the coplanar coil array (b) in a thinly laminated formation with anisotropic behavior.

The coaxial profile will be biased towards the high conductivity laminations (no oil-shale) because the circular induced currents flow parallel to the bedding planes, i.e., the effect of anisotropy is nil because the coaxial signal is due only to the horizontal conductivity $\left(\sigma_{h}\right)$. However, elliptical induced currents from a coplanar array will flow perpendicular to the borehole and bed interfaces, so that polarization charges will accumulate at these boundaries. The coplanar signal is due to both horizontal $\left(\sigma_{h}\right)$ and vertical $\left(\sigma_{v}\right)$ conductivities which $\sigma_{h}$ is usually larger than $\sigma_{v}$ in the clastic sedimentary formations.

Kaufman \& Dashevsky (2003) show that in the low frequency range $(L / \delta \ll 1)$ the quadrature component of the secondary magnetic field (without the mutual term) registered by coplanar array is directly proportional to the vertical conductivity $\left(\sigma_{v}\right)$. For this reason, measuring the ratio of the quadrature components of the coaxial and coplanar arrays allows us to obtain an apparent coefficient of anisotropy $\left(\lambda_{a}^{2}\right)$ which is the same that's 
given by the ratio of the boosted signals of the coplanar and coaxial arrays:

$$
\lambda^{2}=\sigma_{h} / \sigma_{v} \cong Q h_{x}^{c p} / Q h_{z}^{c x}={ }_{c}^{c p} / \sigma_{c}^{c x}=\lambda_{a}^{2}
$$

\section{Results}

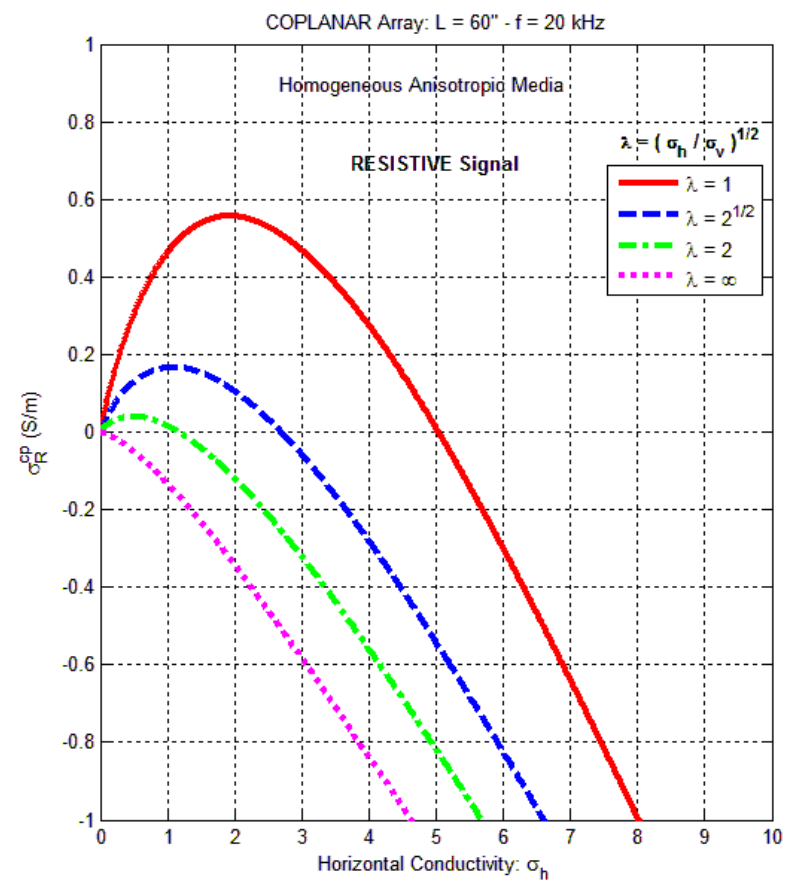

Figure 3 - Coplanar resistive signal $\left(\sigma_{R}^{c p}\right)$ versus horizontal conductivity $\left(\sigma_{h}\right)$ in homogenous anisotropic media $(\lambda=1 ; \sqrt{2} ; 2$ and $\infty)$.

Figure 3 shows the horizontal conductivity on the coplanar resistive signal $\left(\sigma_{R}^{c p}\right)$ in an infinite homogeneous medium. The curves show two opposing tendencies as the horizontal conductivity $\sigma_{h}$ varies: 1) starting at $\sigma_{R}^{c p}=0$ the signals increase with conductivity at low values, and 2) they reaches a peak and then decrease when the skin effect becomes more significant. As the coefficient of anisotropy increases $(\lambda=1 ; \sqrt{2} ; 2$ and $\infty)$, the resistive signal decays gradually showing a strong sensitive to anisotropy.

According Ellis \& Singer (2007), as the conductivity increases, the skin depth decreases and the current lines close around the transmitter so that at the receiver, which is now outside the skin depth, the signal becomes increasingly weaker and shifted in phase. The resistive signal soon reaches a peak and then decreases, eventually going negative when the phase angle is more than 90 。.

Figure 4 shows the coplanar reactive signal without the direct mutual coupling $\left(\sigma_{X F}^{c p}\right)$. It has an opposite behavior of the resistive signal, i.e., the signal decreases as the coefficient of anisotropy increases, partially preserving the information lost in the resistive signal due to skin effect. This out-of-phase component provides a booster to correct the skin effect on the resistive signal in a firstorder approximation, creating a signal $\left(\sigma_{c}\right)$ that has a better fit to the true model $\left(\sigma_{t}\right)$.

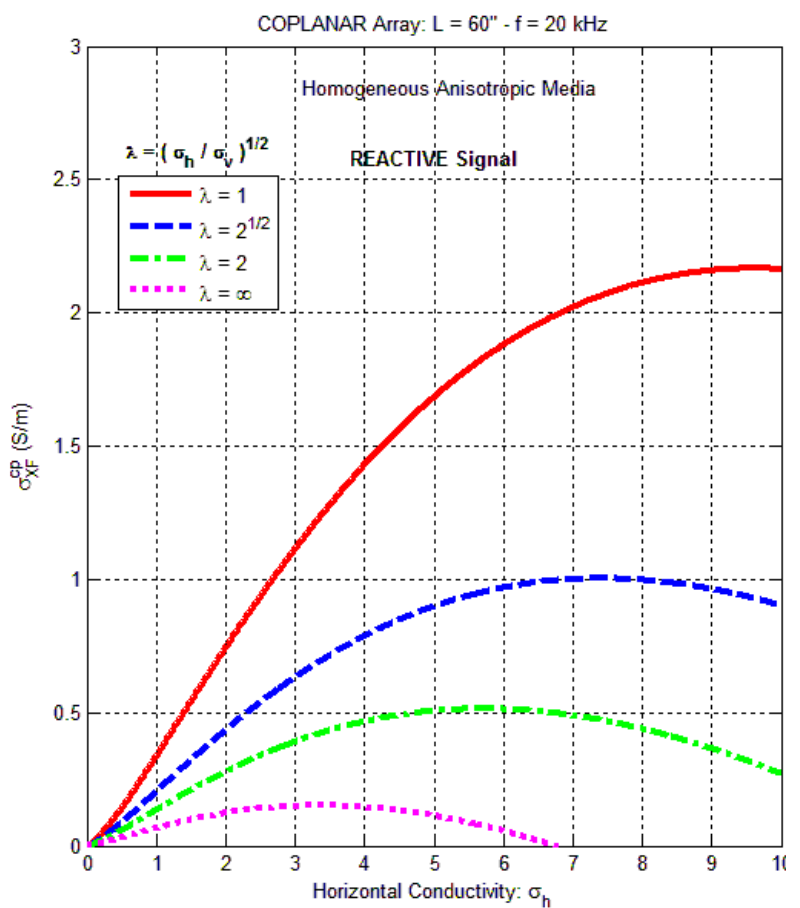

Figure 4 - Coplanar reactive signal $\left(\sigma_{X F}^{c p}\right)$ versus horizontal conductivity $\left(\sigma_{h}\right)$ of the coplanar array $(\mathrm{L}=$ $1.524 \mathrm{~m}$ ) in homogenous anisotropic media.

Figure 5 shows a chart which is entered with resistive ( $\sigma_{R}^{c p}$ ) and reactive ( $\sigma_{X F}^{c p}$ ) signals of the coplanar array it is possible to find the conductivities $\left(\sigma_{h}\right.$ and $\left.\sigma_{v}\right)$ and the coefficient of anisotropy ( $\lambda$ ) to a homogeneous medium. Thus, the coplanar array could be sufficient for measurement of formation anisotropy. However, for nonhomogeneous anisotropic media, the influence of the heterogeneities like bed-boundaries allow us to arrive only at apparent values provides by chart, after an extremely hard and tedious work.

Figure 6 and Figure 7 show the coaxial and coplanar responses, respectively, in a thick package (10.5L) consisting in thinly laminated $(\mathrm{L} / 2)$ formation with low conductivity contrast $\left(\left(\sigma_{\mathrm{sd}}=0.5 \mathrm{~S} / \mathrm{m}\right.\right.$ and $\left.\sigma_{s h}=2.0 \mathrm{~S} / \mathrm{m}\right)$.

The coplanar signals have a more prominent oscillation into the package although suffer a strongest adjacent bed and skin effect. Polarizations "horns" appear in the coplanar profiles, against the package boundaries. These "horns" are more evident on resistive signal. They are caused by the building up of the charges at the boundaries, since the normal component of the electric field is discontinuous at the interfaces. 


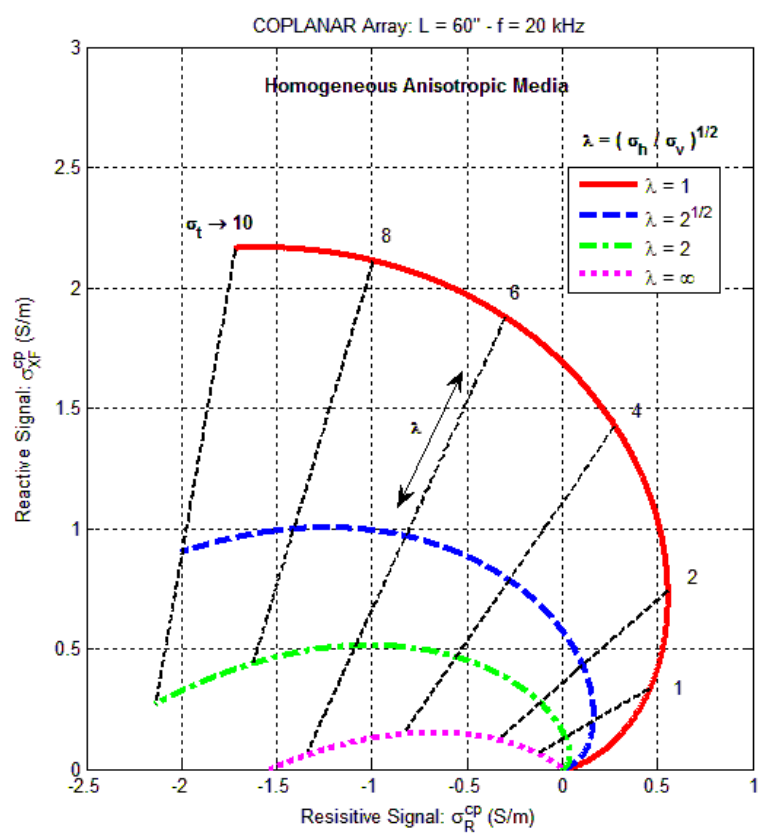

Figure 5 - Coplanar chart to find the conductivities $\left(\sigma_{h}\right.$ and $\left.\sigma_{v}\right)$ and the coefficient of anisotropy $(\lambda)$ to a homogeneous medium..

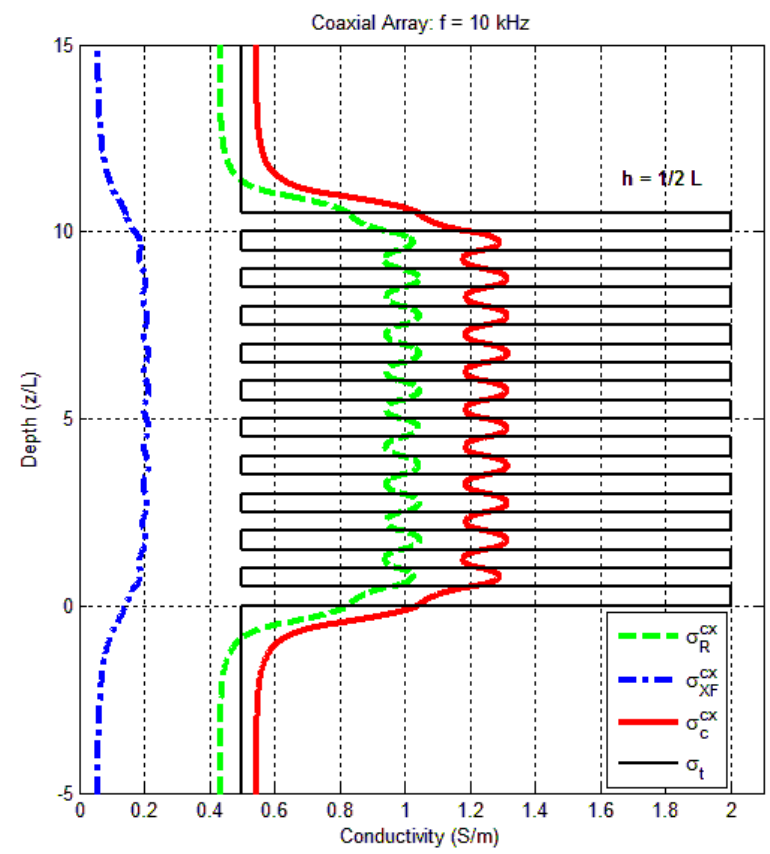

Figure 6 - Resistive $\left(\sigma_{R}^{c x}\right)$, reactive $\left(\sigma_{X F}^{c x}\right)$ and boosted $\left(\sigma_{c}^{c x}\right)$ coaxial signals to a thinly laminated formation.

That build-up of charges acts like a secondary transmitter generating a signal in the proximity of the interfaces. Although, these "horns" may be a good quality bed boundaries indicators, Carvalho \& Verma (1998) showed experimentally, through test tank measurements, that these oscillations on the coplanar responses are damped if the presence of the borehole and invasion are taken into account because these regions acting as a waveguide and accentuating their contributions on the responses. This was one of the reasons that some respected researchers considered the coplanar coil array undesirable.

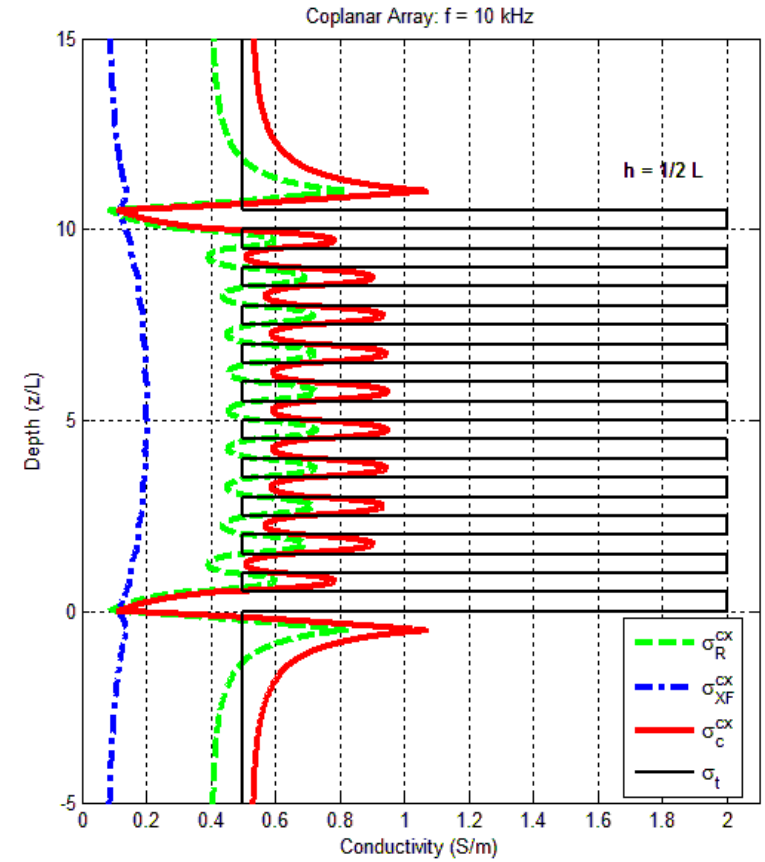

Figure 7 - Resistive $\left(\sigma_{R}^{c p}\right)$, reactive $\left(\sigma_{X F}^{c p}\right)$ and boosted $\left(\sigma_{c}^{c p}\right)$ coplanar signals to a thinly laminated formation $(\mathrm{h}=$ $L$ / 2).

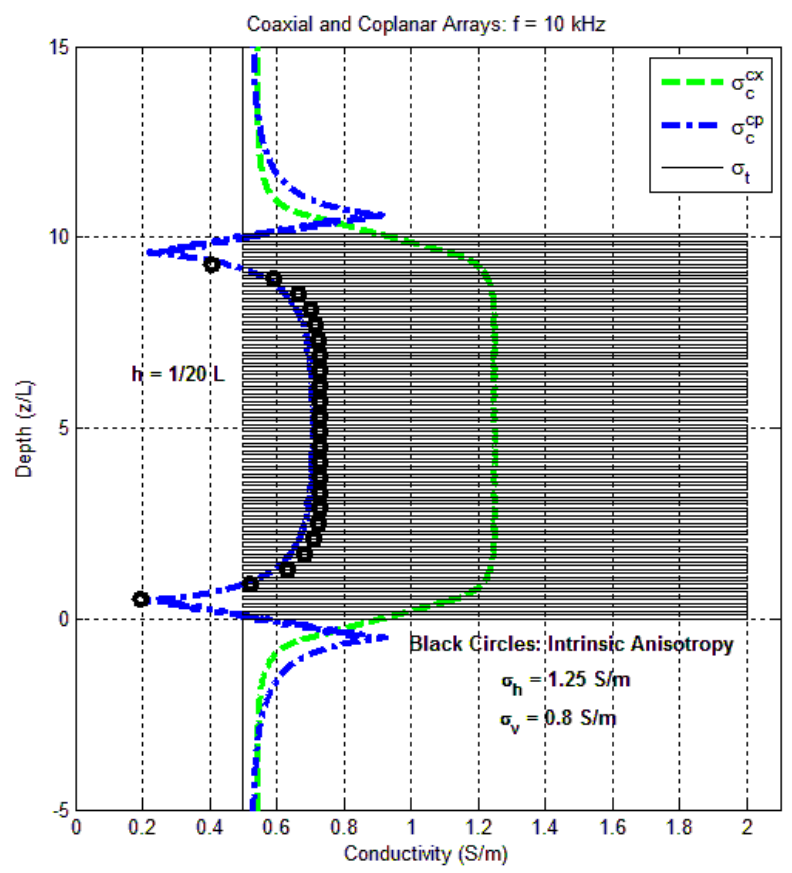

Figure 8 - Coplanar and coaxial boosted signals $\left(\sigma_{c}^{c p}\right.$ and $\left.\sigma_{c}^{c x}\right)$ to a very thinly laminated formation $(\mathrm{h}=\mathrm{L} / 20)$. 
Figure 8 and Figure 9 show the main signals furnished by the modern triaxial induction logging tool, i.e. .the coplanar and coaxial boosted signals $\left(\sigma_{c}^{c p}\right.$ and $\left.\sigma_{c}^{c x}\right)$ and the apparent anisotropy index $\lambda_{a}^{2}$ in a model of thick package, formed by very thinly $(h=L / 20)$ laminated formation.

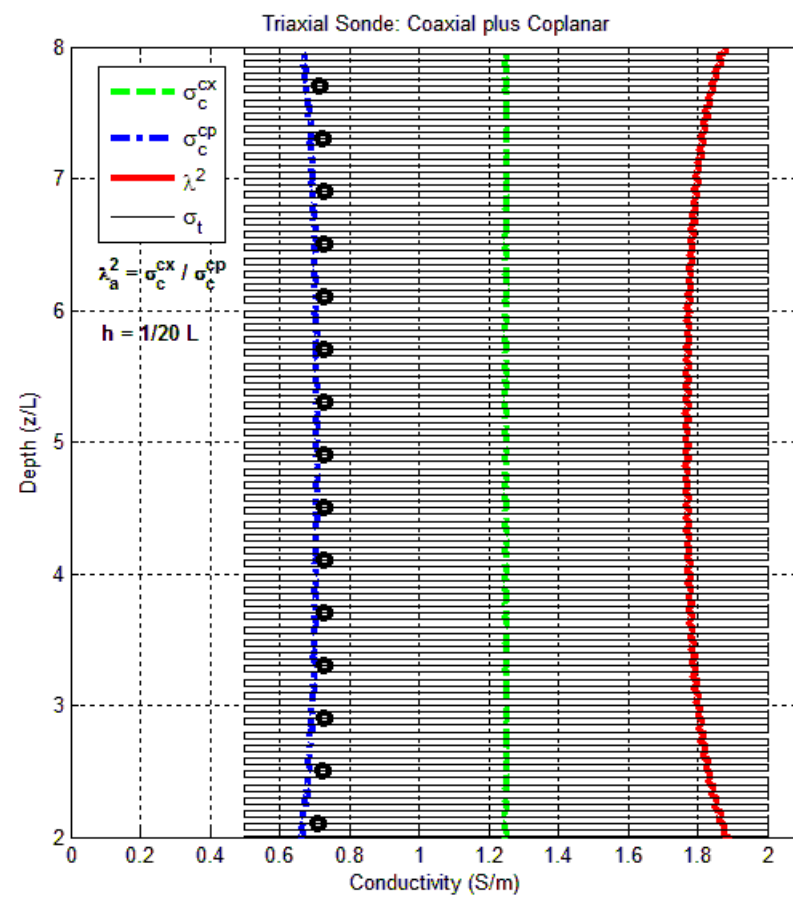

Figure 9 - Boosted signals $\left(\sigma_{c}^{c p}\right.$ and $\sigma_{c}^{c x}$ ) and the anisotropy index $\left(\lambda_{a}^{2}\right)$ of the triaxial tool (coplanar plus coaxial) into a very thinly laminated formation $(h=L /$

Now, we may see that the oscillations of the signals within the laminated package is almost imperceptible due to the thickness of the blades are extremely reduced. In this case, the responses of the laminated package with macroscopy anisotropy tend to converge to the responses of an intrinsically anisotropic layer of the same thickness.

Applying in the Equation (3) the values of $\sigma_{\text {sd }}=0.5 \mathrm{~S} / \mathrm{m}$ and $\sigma_{s h}=2.0 \mathrm{~S} / \mathrm{m}$ we obtain $\sigma_{\mathrm{h}}=1.25 \mathrm{~S} / \mathrm{m}$ and $\sigma_{\mathrm{v}}=0.8$ $\mathrm{S} / \mathrm{m}$. The coplanar response to this intrinsic anisotropy bed is shown by dotted lines (black circles) and we may observe a good agreement with the response of thinly laminated formation.

Through the Equation (4) we obtain to this model a coefficient of anisotropy $\lambda^{2}=1.56$. We may verify a significant difference $(13 \%)$ between this value and the coefficient of anisotropy index shown in Figure 9. It is due: 1 ) the first-order approximation $(\omega \rightarrow 0)$ in the magnetic fields components; 2 ) the adjacent bed effects in the logs and 3) laminae still not thin enough in the model.

Even so, this procedure is consistent with the usual practice in the modern induction logging. Anderson et al. (2008) show this anisotropic index is a useful measurement for determining the level of anisotropy, and when this ratio is higher than five, it alerts the log analyst to look for potential laminated-pay reservoir. A classic example of the literature is in the Krishna-Godavari basin (west coast of India), where the reservoirs were underestimated by more than $60 \%$ by traditional induction tools, which used only coaxial array.

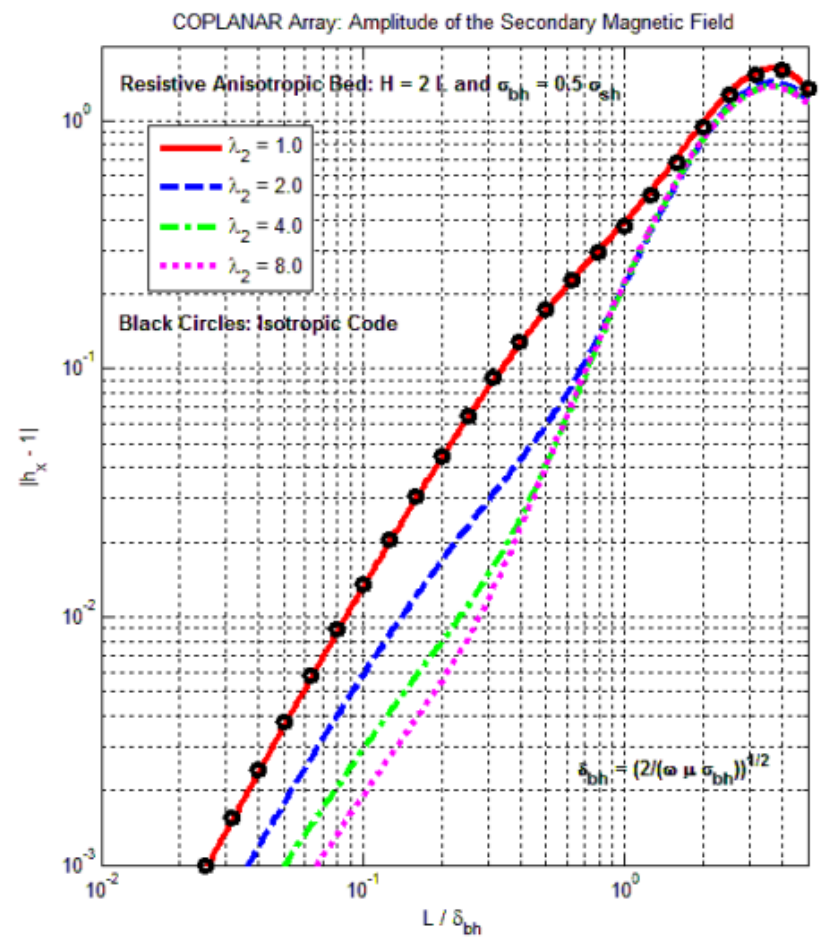

Figure 10 - Amplitude of the secondary magnetic field within an anisotropic resistive bed.

Figure 10 and Figure 11 show the magnitude of the secondary magnetic field normalized by direct mutual coupling $\left(\left|h_{x}^{c p}-1\right|\right)$ versus the coil spacing normalized by skin effect within an anisotropic bed $\left(L / \delta_{b h}\right)$ which has a relative thickness $H=2 L$ when it is more resistive $\left(\sigma_{b}=\right.$ $\left.0.5 \sigma_{s}\right)$ or conductive $\left(\sigma_{b}=2 \sigma_{s}\right)$ than the surrounding medium, respectively. In both cases we show too the isotropic responses (black circles) to validate our anisotropic code. As is seen from the curves, the influence of the coefficient of anisotropy of the bed on the amplitude responses is significant at the range of relatively low frequencies $\left(L / \delta_{b h}<0.5\right)$ and the influence of change of conductivity exceeds the influence due to a change of anisotropy coefficient of the bed.

\section{Conclusion}

In this paper, we showed some numerical responses for one-dimensional (1D) layered anisotropic models, in which the presence of the borehole and the invasion zones are neglected, to simulate geological environments of hydrocarbon reservoirs with intrinsic electrical anisotropy.

We ignore the six cross-coupled components because our models have azimuthal symmetry which these signals 
are nulls. Thus, we modeled only the responses obtained from the coaxial and coplanar coil arrays, this leaves us with two different components of the magnetic field: $H_{z z}$ to the coaxial and $H_{x x}=H_{y y}$ to the coplanar responses.



Figure 11 - Amplitude of the secondary magnetic field within a anisotropic conductive bed.

The results show a strong sensitivity of the coplanar array to skin effect and above all a good sensitivity to anisotropy of the formations.

Our main goal was to compare the responses with those from isotropic finely laminated zones and to perform a quantitative analysis of how well an intrinsic anisotropic layer can represent such environments within a chosen tolerance.

As is seen from the curves, the influence of the coefficient of anisotropy is significant at the range of relatively low frequencies $\left(L / \delta_{b h}<0.5\right)$ and the influence of change of conductivity exceeds the influence due to a change of anisotropy coefficient of the bed.

\section{Acknowledgments}

Paulo Carvalho thanks the CAPES (Coordenação de Aperfeiçoamento de Pessoal de Nível Superior) for the post-doctoral fellowship in the "Programa de PósGraduação em Geofísica" of the Federal University of Pará (CPGf/UFPA).

\section{References}

ANDERSON B., BARBER T, BASTIA R, CLAUVAUD JB, COFFIN B, DAS M, HAYDEN R, KLIMENTOS T, MINH CC \& WILLIAMS S. 2008. Triaxial induction - A new angle for an old measurement. Oilfied Review.

CARVALHO, de P. R. \& VERMA, O. P. 1998. Induction tool with a coplanar coil system. The Log Analyst, 39 (6): 48-53.

CARVALHO, de P. R.; RÉGIS, C. T. R. \& HOWARD, A. Q. Jr. 2012. Coaxial and coplanar arrays in the triaxial induction tools: dipole and loop models. V Simpósio Brasileiro de Geofísica, Salvador, Bahia.

DOLL, H. G. 1949. Introduction to induction logging and application to logging of wells drilled with oil-based mud. Journal of Petroleum Technology.

ELLIS D.V. \& SINGER J.M. 2007. Well logging for earth scientists. $2^{\text {nd }}$ ed. Springer. 692 pp.

KAUFMAN, A. A. \& DASHEVSKY, Y. A. 2003. Principles of induction logging. Amsterdam Elsevier Publishers.

KRIGSHÄUSER B, FANINI O, FORGANG S, ITSKOVICH G, RABINOVICH M, TABAROVSKY L. \& YU L. 2000. A new multicomponent induction logging tool to resolve anisotropic formations: 41st Ann. Logging Sym., Soc. Prof. Well Log. Analysts, paper D.

OMERAGIC D., BAYRAKTAR Z., THIEL M., HABASHY, T., ALATRACH S. \& SHRAY F. 2015. Triaxial Induction Interpretation in Horizontal Wells: Mapping Boundaries, Characterizing Anisotropy and Fractures. In: Annual Logging Symposium, 56th. Long Beach, California, USA. Anais SPWLA. 